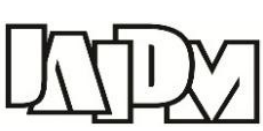

Journal of $\boldsymbol{A I}$ and Data Mining

Vol 3, No 1, 2015, 113-120.

doi:10.5829/idosi.JAIDM.2015.03.01.12

\title{
Discrete time robust control of robot manipulators in the task space using adaptive fuzzy estimator
}

\author{
M. M. Fateh* and S. Azargoshasb \\ Electrical Engineering Department, University of Shahrood, Shahrood, Iran. \\ Received 07 March 2014; Accepted 21 September 2014 \\ *Corresponding author: mmfateh@shahroodut.ac.ir (M. M. fateh).
}

\begin{abstract}
This paper presents a discrete-time robust control for electrically driven robot manipulators in the task space. A novel discrete-time model-free control law is proposed by employing an adaptive fuzzy estimator for the compensation of the uncertainty including model uncertainty, external disturbances and discretization error. Parameters of the fuzzy estimator are adapted to minimize the estimation error using a gradient descent algorithm. The proposed discrete control is robust against all uncertainties as verified by stability analysis. The proposed robust control law is simulated on a SCARA robot driven by permanent magnet de motors. Simulation results show the effectiveness of the control approach.
\end{abstract}

Keywords: Discrete Control, Uncertainty Estimator, Free-model Control, Adaptive Fuzzy Estimator, Taskspace, SCARA Robot.

\section{Introduction}

The Advantages of digital systems to analogue systems lead us to pay more attention on developing the discrete control theory and using digital controllers. Digital control systems are superior to continuous time control systems from different points of view. For instance, digital systems are more flexible to changes, more immune to environmental noises and less computational [1]. The stability analysis of discrete control system has been rigorously studied. Stability analysis of discrete-time fuzzymodel-based control systems with time delays has been introduced in [2].was presented [2]. Stability analysis and stabilization of discrete-time T-S fuzzy time-varying delay systems has been studied in [3]. A model reference adaptive control approach for the synchronization of a discretetime chaotic system is using output tracking control has been performed [4]. The important purposes in these researches are the improvement of the control performance and to ensure the system stability at the presence of problems such as uncertainties, nonlinearities, sampling period and discretizing errors. Discrete control of robot manipulators has achieved a great deal of research in various forms of control algorithms. The discrete repetitive linear controls namely Q-filter, convolution, learning and basis function were offered and compared in [5]. Among them, the Qfilter algorithm as an internal control shows the fastest execution speed, the lowest computational complexity, and ease of design and implementation. However, tracking errors cannot converge to zero due to nonlinearity of the robotic system. Many research efforts have paid attention to the model-based control. However, a precise model is not available in practice. In addition, complex models may not be used in the control laws for avoiding computational burden and practical difficulties. Therefore, a simpler model such as nominal model is preferred to design a controller. As a result, the control system faces uncertainty raised from differences between the nominal model and actual system. Generally, the uncertainty may include the parametric uncertainty, unmodeled dynamics, external disturbances and discretization error. The control performance is thus dependent on how well the uncertainty can be estimated and compensated. In order to overcome model uncertainties, a discrete sliding-mode control was developed for robot manipulators [6]. Another approaches model-free 
discrete control for uncertain robot manipulators was presented using a fuzzy estimator [7]. Alternatively, this paper presents a model-free control by estimation of the uncertainty. In the last few decades, adaptive and robust control of robot manipulators in the task-space [8-9] and jointspace [10] have been extensively studied. The task-space control is much complicated than the joint-space control in the presence of uncertainties. Among the control strategies used for the robot manipulators, the torque control strategy is more complicated than the voltage control strategy [11]. Position control of flexible joint robots using torque control strategy is a challenging task, while voltage control strategy has considerably simplified this problem [12-13].

Nowadays, various fuzzy systems are widely used in adaptive and robust control of robot manipulators [14-15] due to their universal approximation property [16]. These researches can be considered as different efforts made towards a common objective which is estimation and compensation of uncertainty. For example, using the $\mathrm{T}-\mathrm{S}$ model of discrete-time chaotic systems, an adaptive control algorithm was presented based on some conventional adaptive control methods [17]. Another approach based on adaptive fuzzy sliding mode control has been presented for the position control of robotic manipulators [18]. Most learning algorithms such as the gradient descent algorithm require some training data for estimation a function as a fuzzy system or a neural network for example. In estimation of uncertainty, the problem with this procedure is that the training data is not available unknown. To solve this problem, this paper finds a relationship between the tracking error and the estimation error to perform the gradient descent algorithm. Thus, the need for providing training data will be eliminated. One of the most challenging problems in designing a robust controller is to employ an uncertainty bound parameter which should be known in advance or estimated [19-20]. The value of uncertainty bound parameter is very crucial. Overestimation of this parameter will result in saturation of input and higher frequency of chattering in the switching control laws, while underestimation will increase the tracking error [21]. By estimation the uncertainty, a new robust control approach is proposed which is free from the uncertainty bound parameter. In this paper, a discrete-time robust adaptive fuzzy control has been proposed for position control of a robot manipulator using voltage control strategy in task space. In the proposed method, the consequence parts of fuzzy rules are tuned via adaptive laws using a gradient descent algorithm. Most of robust control approaches are based on linear parameterization property of the manipulator dynamic equation. Consequently, the regressor matrix should be identified which requires a tedious procedure. However, the model free control approach presented in this paper does not need the regressor analysis. As result, it is much simpler and less computational. This paper is organized as follows: Section 2 introduces modeling of the electrical robot manipulator driven by geared permanent magnet dc motors. Section 3 presents the proposed discrete control law. Section 4 describes the discrete adaptive fuzzy method to estimate and compensate the uncertainties. Section 5 deals with stability analysis and performance evaluation. Section 6 presents the simulation results and finally, section 7 concludes the paper.

\section{Modeling}

Consider an electrical robot driven by geared permanent magnet dc motors. The dynamics of robot manipulator [22] is expressed as

$$
\mathbf{D}(\mathbf{q}) \ddot{\mathbf{q}}+\mathbf{C}(\mathbf{q}, \dot{\mathbf{q}}) \dot{\mathbf{q}}+\mathbf{G}(\mathbf{q})=\boldsymbol{\tau}
$$

where, $\mathbf{q} \in R^{n}$ is the vector of joint positions, $\mathbf{D}(\mathbf{q}) \in R^{n \times n}$ the inertia matrix of manipulator, $\mathbf{C}(\mathbf{q}, \dot{\mathbf{q}}) \dot{\mathbf{q}} \in R^{n}$ the vector of centrifugal and Coriolis torques, $\mathbf{G}(\mathbf{q}) \in R^{n}$ the vector of gravitational torques, and $\tau \in R^{n}$ the vector of joint torques. The vectors and matrices are shown bold for clarity. We assume that the mechanical system is perfectly rigid. The electric motors provide the joint torques $\tau$ by

$$
\ddot{\mathbf{\theta}}_{\mathrm{m}}+\mathbf{B} \dot{\boldsymbol{\theta}}_{\mathrm{m}}+\mathbf{r} \boldsymbol{\tau}=\boldsymbol{\tau}_{\mathrm{m}}
$$

where, $\tau_{\mathrm{m}} \in R^{n}$ is the torque vector of motors, $\boldsymbol{\theta}_{\mathrm{m}} \in R^{n}$ the position vector of motors, and $\mathbf{J}, \mathbf{B}, \mathbf{r} \in R^{n \times n}$ the diagonal matrices for inertia, damping, and reduction gear of motors, respectively. The vector of joint velocities $\dot{\mathbf{q}}$ is obtained by the vector of motor velocities $\dot{\boldsymbol{\theta}}_{\mathbf{m}}$ through the gears as

$$
\mathbf{r} \dot{\boldsymbol{\theta}}_{\mathrm{m}}=\dot{\mathbf{q}}
$$

Note that vectors and matrices are represented in the bold form for clarity. In order to obtain the motor voltages as the inputs of system, consider the electrical equation of geared permanent magnet dc motors in the matrix form as 


$$
\mathbf{R} \mathbf{I}_{\mathbf{a}}+\mathbf{L} \dot{\mathbf{I}}_{\mathbf{a}}+\mathbf{K}_{\mathrm{b}} \mathbf{r}^{-1} \dot{\mathbf{q}}=v
$$

where, $v \in R^{n}$ is the vector of motor voltages, and $\mathbf{I}_{\mathrm{a}} \in R^{n}$ the vector of motor currents. $\mathbf{R}, \mathbf{L}, \mathbf{K}_{\mathbf{b}} \in R^{n \times n}$ represent the diagonal matrices for the armature resistance, inductance, and back-emf constant of the motors, respectively. The motor torque vector $\boldsymbol{\tau}_{\mathbf{m}}$ as the input for the dynamic (2) is produced by the motor current vector

$$
\mathbf{K}_{\mathrm{m}} \mathbf{I}_{\mathrm{a}}=\boldsymbol{\tau}_{\mathrm{m}}
$$

where, $\mathbf{K}_{\mathbf{m}}$ is the diagonal matrix of the torque constants. A model for the electrically driven robot in the state space is introduced by the use of (1)-(5) as

$$
\dot{\mathbf{x}}=\mathbf{f}(\mathbf{x})+\mathbf{b v}
$$

Where

$$
\begin{aligned}
& \mathbf{f}(\mathbf{x})=\left[\begin{array}{c}
\mathbf{x}_{2} \\
\left(\mathbf{J r} \mathbf{r}^{-1}+\mathbf{r D}\left(\mathbf{x}_{1}\right)\right)^{-1}\left(-\left(\mathbf{B r}^{-1}+\mathbf{r} \mathbf{C}\left(\mathbf{x}_{1}, \mathbf{x}_{2}\right)\right) \mathbf{x}_{2}-\mathbf{r G}\left(\mathbf{x}_{1}\right)+\mathbf{K}_{\mathbf{m}} \mathbf{x}_{3}\right) \\
-\mathbf{L}^{-1}\left(\mathbf{K}_{\mathbf{b}} \mathbf{r}^{-1} \mathbf{x}_{2}+\mathbf{R} \mathbf{x}_{3}\right)
\end{array}\right] \\
& \mathbf{b}=\left[\begin{array}{c}
\mathbf{0} \\
\mathbf{0} \\
\mathbf{L}^{-1}
\end{array}\right], \mathbf{x}=\left[\begin{array}{c}
\mathbf{q} \\
\dot{\mathbf{q}} \\
\mathbf{I}_{\mathbf{a}}
\end{array}\right]
\end{aligned}
$$

The state space (6) is a nonlinear multivariable multi-input/multi-output system. The complexity of the model (6) faces serious challenges in the field of robot control. In order to consider the actuator dynamics, the voltages of motors denoted by $\mathbf{V}$ were considered as the inputs of the robotic system (6). To simplify the control problem, the majority of the control approaches have not considered the actuator dynamics by using the joint torques $\boldsymbol{\tau}$ as the inputs of the system. However, the control performance will be degraded in the high-speed application. Another issue is that the previous control approaches presented for robot manipulators have been based on the second order dynamics of the robot manipulator in a companion form whereas the model (6) is neither the second order nor the companion form.

\section{Proposed discrete robust adaptive fuzzy control}

From the matrix (4), the voltage equation for the ith motor can be written in the scalar form as

$$
R I_{a}+L \dot{I}_{a}+K_{b} r^{-1} \dot{q}+\varphi=v
$$

The variable $\varphi$ denotes the external disturbance. Equation (8) includes both the input $v$ and the joint velocity $\dot{q}$, thus can be considered for position control of the robot manipulator. With the advantage of simplicity, it is preferred for control purposes compared to the complex system (6). The task space velocity $\dot{X}(t)$ is related to joint space velocity $\dot{q}(t)$ as [9]

$\dot{X}=J(q) \dot{q}$

where, $J(q) \in R^{n \times n}$ is the Jacobian matrix from joint space to task space. The derivative of (9) respect to time can be written as

$\ddot{X}=J(q) \ddot{q}+\dot{J}(q) \dot{q}$

$\dot{J}(q)$ exists if the desired path is smooth. Assuming there are no singular points in the desired path in task space such that the Jacobian matrix is of full rank. One can easily obtain from (9) that $\dot{q}=J^{-1}(q) \dot{X}$.

In this paper, it us assumed that the manipulator operates in a region where $J^{-1}(q)$ is nonsingular. Thus, the voltage (8) can be rewritten as

$R I_{a}+L \dot{I}_{a}+K_{b} r^{-1} J^{-1}(q) \dot{X}+\varphi=v$

Equation (11) can be rewritten as

$\hat{J}^{-1} \ddot{X}+g=v$

where, $\hat{J}^{-1}$ is an estimate and inverse of $J$, and $g$ is expressed as

$g=R I_{a}+L \dot{I}_{a}+K_{b} r^{-1} J^{-1} \dot{X}+\varphi-\hat{J}^{-1} \ddot{X}$

In order to propose a model-free controller, one can consider the system (12) in which $g$ is referred to as the uncertainty. One can obtain from (12) a linear discrete system using a sampling period $T$ that is a small positive constant. Substituting $k T$ into $t$ for $k T \leq t<(k+1) T$ and $k=1,2, \ldots$ the discrete time model of the system can be given by

$\hat{J}_{k}^{-1} \ddot{X}_{k}+g_{k}+\varepsilon_{1}=v$

where, $\quad \ddot{X}_{k}=\ddot{X}(k T), \quad \hat{J}_{k}^{-1}=\hat{J}^{-1}(k T) \quad$ and $g_{k}=g(k T)$ and the discretizing error $\varepsilon_{1}$ is expressed as

$\varepsilon_{1}=\hat{J}^{-1} \ddot{X}-\hat{J}_{k}^{-1} \ddot{X}_{k}+g-g_{k}$ 
A discrete adaptive fuzzy control law is proposed as

$$
\hat{J}_{k}^{-1}\left(\ddot{X}_{d, k}+k_{d} \dot{e}_{k}+k_{p} e_{k}\right)+f_{k}=v
$$

where, $v$ is the output of the controller which is given to the voltage input of the motor. $e_{k}=X_{d, k}-X_{k}$ is defined as the tracking error, $\dot{e}_{k}=\dot{X}_{d, k}-\dot{X}_{k}$ is derivative the tracking error of $e_{k}$, and $k_{p}$ and $k_{d}$ are the control design parameters. The term $f_{k}$ is an adaptive fuzzy system for compensating uncertainty. $\ddot{X}_{d, k}$ denotes the discrete-time value of the desired joint acceleration $\ddot{X}_{d}(t)$ using a zero order hold converter defined as

$$
\begin{gathered}
\ddot{X}_{d, k}=\ddot{X}_{d}(k T) \quad \text { for } k T \leq t<(k+1) T \\
\text { and } k=0,1,2 \ldots
\end{gathered}
$$

\section{Discrete adaptive fuzzy estimation of the uncertainty}

Applying control law (16) to the discrete-time model of the system (14) obtains the closed loop system,

$\hat{J}_{k}^{-1}\left(\ddot{e}_{k}+k_{d} \dot{e}_{k}+k_{p} e_{k}\right)=g_{k}+\varepsilon_{1}-f_{k}$

where, $\ddot{X}_{d, k}-\ddot{X}_{k}=\ddot{e}_{k}$, (18) can be written as

$\hat{J}_{k}^{-1}\left(\ddot{e}_{k}+k_{d} \dot{e}_{k}+k_{p} e_{k}\right)=G_{k}-f_{k}$

where,

$G_{k}=g_{k}+\varepsilon_{1}$

Suppose that $f_{k}$ is the output of an adaptive fuzzy system with inputs of $e_{k}, \dot{e}_{k}$ and $\ddot{e}_{k}$. If two fuzzy sets are given to each fuzzy input, the whole control space will be covered by eight fuzzy rules. The linguistic fuzzy rules are proposed in the Mamdani type of the form

$$
\begin{aligned}
& F R_{l} \text { : if } e_{k} \text { is } A_{1}^{l} \text { and } \dot{e}_{k} \text { is } A_{2}^{l} \text { and } \ddot{e}_{k} \text { is } A_{3}^{l} \\
& \text { then } f_{k}=C_{l}
\end{aligned}
$$

where, $F R_{l}$ denotes the lth fuzzy rule for $l=1, \ldots, 8$. In the lth rule $A_{1}^{l}, A_{2}^{l}, A_{3}^{l}$ and $C_{l}$ are the fuzzy membership functions belonging to the fuzzy variables $e_{k}, \quad \dot{e}_{k}, \quad \ddot{e}_{k}$ and $f_{k}$ respectively. Two Gaussian membership functions named as Positive $(\mathrm{P})$ and Negative $(\mathrm{N})$ are defined for the input $e_{k}$ in the operating range of manipulator as shown in figure 1. The same membership functions as for $e_{k}$ are assigned to $\dot{e}_{k}$ and $\ddot{e}_{k}$. Eight symmetric Gaussian membership functions are defined for $f_{k}$ in the form of

$$
\mu_{l}\left(f_{k}\right)=\exp \left(-\left(\frac{f_{k}-\bar{y}_{k}^{l}}{\sigma}\right)^{2}\right) \text { for } l=1, \ldots, 9
$$

where, $\sigma$ and $\bar{y}_{k}^{l}$ are the design parameters. $\sigma$ is constant whereas $\bar{y}_{k}^{l}$ is adjusted by an adaptive law.

The fuzzy rules should be defined such that the tracking control system goes to the equilibrium point. We may use an expert's knowledge, the trial and error method, or an optimization algorithm to design the fuzzy controller. The obtained fuzzy rules are given in table 1 in [7]. In this paper, $f_{k}$ is adapted using a gradient descent algorithm to minimize the tracking error.

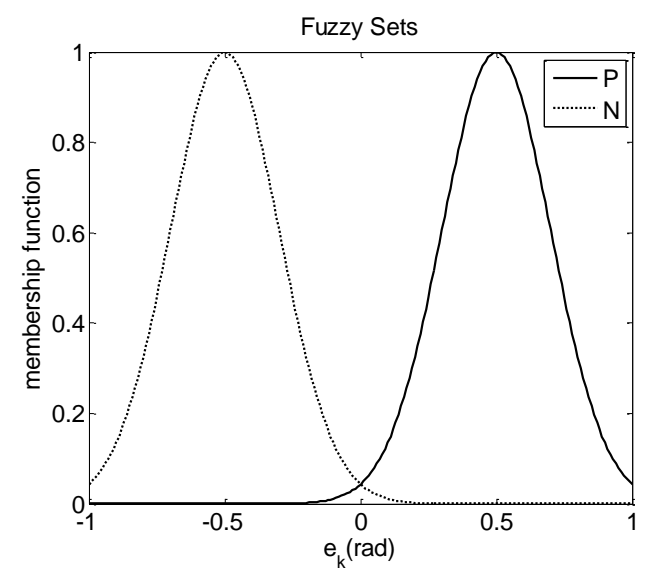

Figure 1. Membership functions of the input $e_{k}$.

If we use the product inference engine, singleton fuzzifier, center average defuzzifier, and Gaussian membership functions, the fuzzy system [23] is of the form

$f_{k}\left(e_{k}, \dot{e}_{k}, \ddot{e}_{k}\right)=\frac{\sum_{L=1}^{8} \bar{y}_{k}^{l} z_{k}^{l}}{\sum_{L=1}^{8} z_{k}^{l}}$

where,

$z_{k}^{l}=\mu_{A_{1}^{l}}\left(e_{k}\right) \mu_{A_{2}^{l}}\left(\dot{e}_{k}\right) \mu_{A_{3}^{l}}\left(\ddot{e}_{k}\right)$

where, $\quad \mu_{A_{1}^{l}}\left(e_{k}\right) \in[0,1], \quad \mu_{A_{2}^{l}}\left(\dot{e}_{k}\right) \in[0,1] \quad$ and $\mu_{A_{3}^{l}}\left(\ddot{e}_{k}\right) \in[0,1]$ are the membership functions for the fuzzy sets $\mu_{A_{1}^{l}}, \mu_{A_{2}^{l}}$ and $\mu_{A_{3}^{l}}$ respectively, and $\bar{y}_{k}^{l}$ is the center of fuzzy set $C_{l}$. The objective is to design a fuzzy system $f_{k}$ so that the estimation error is minimized. 


$$
E_{k}=\frac{1}{2}\left(G_{k}-f_{k}\right)^{2}
$$

The parameter which should be adjusted online is $\bar{y}_{k}^{l}$. The adaptation law in the gradient descent algorithm is given by [26]

$$
\bar{y}_{k+1}^{l}=\bar{y}_{k}^{l}-\alpha \frac{\partial E_{k}}{\partial \bar{y}_{k}^{l}}
$$

where, $\alpha$ is a positive constant which determines the speed of convergence and $\frac{\partial E_{k}}{\partial \bar{y}_{k}^{l}}$ is calculated

as

$$
\frac{\partial E_{k}}{\partial \bar{y}_{k}^{l}}=-\frac{z_{k}^{l}\left(G_{k}-f_{k}\right)}{\sum_{L=1}^{9} z_{k}^{l}}
$$

Note that $G_{k}$ is an unknown function. So it is unavailable and cannot be used in the adaptation law. To solve the problem, this paper proposes a novel technique. Substituting (19) into (27) yields to

$$
\frac{\partial E_{k}}{\partial \bar{y}_{k}^{l}}=-\frac{z_{k}^{l}\left(\hat{J}_{k}^{-1}\left(\ddot{e}_{k}+k_{d} \dot{e}_{k}+k_{p} e_{k}\right)\right)}{\sum_{L=1}^{9} z_{k}^{l}}
$$

The proposed model (14) is purposeful. The used technique for calculating $\frac{\partial E_{k}}{\partial \bar{y}_{k}^{l}}$ in (28) for the gradient descent algorithm implies that the algorithm does not require data as input-output pairs for the unknown function. Substituting (28) into (26) yields to adaptive rule

$$
\bar{y}_{k+1}^{l}=\bar{y}_{k}^{l}+\alpha \frac{z_{k}^{l}\left(\hat{J}_{k}^{-1}\left(\ddot{e}_{k}+k_{d} \dot{e}_{k}+k_{p} e_{k}\right)\right)}{\sum_{L=1}^{9} z_{k}^{l}}
$$

\section{Stability}

A proof for the boundedness of the state variables $\mathbf{q}, \dot{\mathbf{q}}$ and $\mathbf{I}_{\mathbf{a}}$ is given by stability analysis. In order to analyze the stability, the following assumptions are made:

Assumption 1 The desired trajectory $X_{d, k}$ must be smooth in the sense that $X_{d, k}$ and its derivatives up to a necessary order are available and all uniformly bounded [22].

As a necessary condition to design a robust control, the external disturbance must be bounded. Thus, the following assumption is made:

Assumption 2 The external disturbance $\varphi$ is bounded as $|\varphi(t)| \leq \varphi_{\max }$.
Assumption 3 we assume that the robot is operating in a finite task space such that the Jacobian matrix is full rank. Assuming there are no singular points in the desired path in task space such that the inverse of Jacobian matrix, $\hat{J}_{k}^{-1}$ is bounded.

The closed loop system (19) can be represented as $\hat{J}_{k}^{-1}\left(\ddot{e}_{k}+k_{d} \dot{e}_{k}+k_{p} e_{k}\right)=w$

where, $w=G_{k}-f_{k}$. The linear second order differential (30) with $k_{p}>0, k_{d}>0$ and the boundedness of $\hat{J}_{k}^{-1}$ is stable based on the RouthHorwitz criteria. The output $e_{k}$ is bounded if the input $w$ be bounded. The gradient descent algorithm obtains the reduction of error expressed by $E_{k}=\frac{1}{2}\left(G_{k}-f_{k}\right)^{2} \quad$ in (25). Therefore, $w=G_{k}-f_{k}$ is bounded. In the system (30), the boundedness of input $w$ implies that

Result 1. $e_{k}, \dot{e}_{k}$ and $\ddot{e}_{k}$ are bounded.

We have $X_{k}=X_{d, k}-e_{k}$. According to the Assumption 1, $X_{d, k}$ is bounded. Thus,

Result 2. The task space position $X_{k}$ is bounded. Since $\mu_{A_{1}^{l}}, \mu_{A_{2}^{l}}, \mu_{A_{3}^{l}} \in\left[\begin{array}{ll}0 & 1\end{array}\right]$, thus according to (24) one can imply that $\left|z_{k}^{l}\right| \leq 1$. Thus,

Result 3. The function $z_{k}^{l}$ is bounded.

Parameter $\bar{y}_{k+1}^{l}$ was expressed as (29), in which, parameters $k_{p}$ and $k_{d}$ are constant, and $\hat{J}_{k}^{-1}$ is bounded. As expressed in Result $1, e_{k}, \dot{e}_{k}$ and $\ddot{e}_{k}$ are bounded. The boundedness of $z_{k}^{l}$ was given in Result 3. Therefore,

Result 4. The parameter $\bar{y}_{k+1}^{l}$ is bounded.

From (23), Result 3 and Result 4, it can be concluded that

Result 5. Function $f_{k}$ is bounded.

Considering control law (16) and using Assumption 1 and 3 for boundedness of $\ddot{X}_{d, k}$ and $\hat{J}_{k}^{-1}$, Result 5 for boundedness of $f_{k}$, and Result 1 for boundedness of $e_{k}$ and $\dot{e}_{k}$ verifies that

Result 6. Motor voltage $v$ is bounded.

According to [13], in an electrically driven robot when the motor voltage is bounded, it is proven that

Result 7. The motor velocity $\dot{q}$, and the motor current $I_{a}$ are bounded.

Since 
$q=\int_{0}^{t} \dot{q} d t$

As expressed in Result 7, $\dot{q}$ is bounded, and according to boundedness $t$, Therefore

Result 8. , The joint position $q$ is bounded.

As a result, the joint position $q$ in Result 8 , the joint velocity $\dot{q}$, and the motor current $I_{a}$ in Result 7 are bounded. Applying this reasoning for all joints imply that

Result 9. The state vectors $\mathbf{q}, \dot{\mathbf{q}}$, and $\mathbf{I}_{\mathbf{a}}$ are bounded.

Therefore, the stability is proven.

\section{Simulation results}

The control law (16) is simulated using a three link SCARA robot driven by permanent magnet $\mathrm{dc}$ motors presented by [24]. The parameters of motors are given in table 2 in [7]. In the simulations, the arm which consists of the first three joints is used to perform the proposed taskspace control law. The fourth joint is locked. The maximum voltage of each motor is set to $v_{\max }=40$.

Simulation 1 In this simulation, the desired trajectory in the task-space is defined as

$$
\boldsymbol{X}_{d}=\left[0.75-0.1 \cos \left(\frac{\pi k T}{3}\right) ; 0.65-0.1 \sin \left(\frac{\pi k T}{3}\right) ; 0\right]^{T}
$$

To consider the kinematical uncertainties, $\hat{J}(q)$ is selected as $\hat{J}(q)=0.8 J(q)$. The adaptation rule (29) is set to $\bar{y}_{k}^{l}(0)=0, \alpha=0.285, k_{d}=108$ and $k_{p}=388$. The external disturbance is given zero.

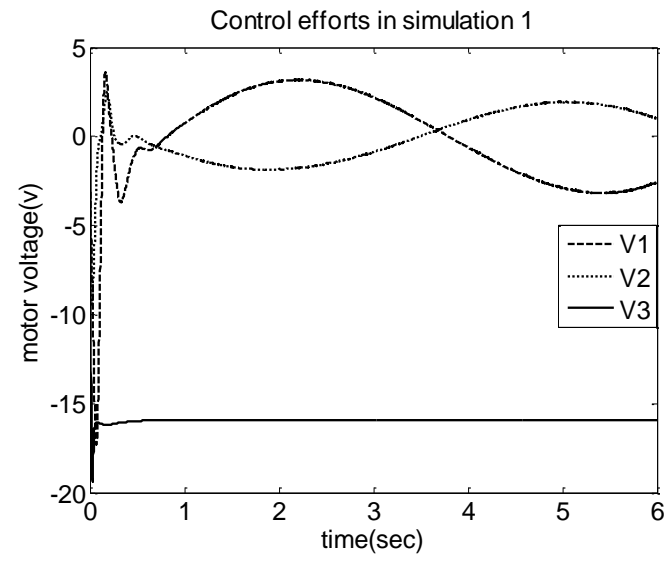

Figure 2. The control efforts in simulation 1.

Figure 2 illustrates the control efforts which are satisfactory. The motor voltages are smooth and under the maximum permitted voltage. The tracking performance in the $x y$ plane and along the $z$ axis are shown in figure 3 and figure 4, respectively. The tracking performance confirm that the parameters are well adapted as shown in figure 5 .

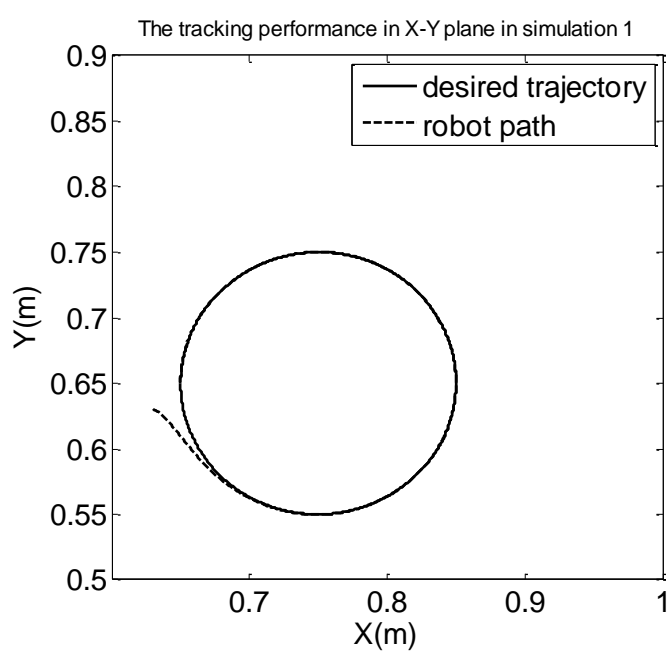

Figure 3. The tracking performance in the $x y$ plane.

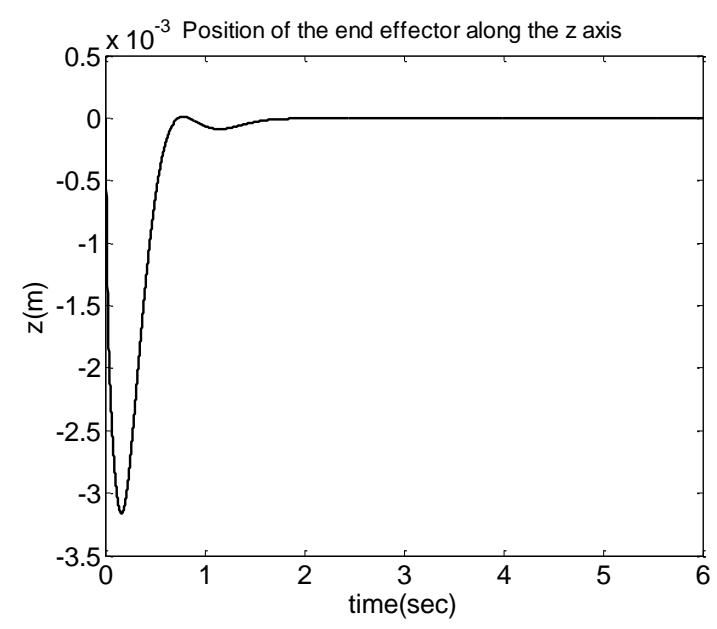

Figure 4. The tracking performance along the $z$ axis.

Simulation 2: In this simulation, the desired trajectory in the task-space is defined as (33). To consider the kinematical uncertainties, $\hat{J}(q)$ is selected as $\hat{J}(q)=0.8 J(q)$. The adaptation rule (29) is set to $\bar{y}_{k}^{l}(0)=0, \alpha=0.29, k_{d}=100$ and $k_{p}=380$. The external disturbance is inserted to the input of each motor as a periodic pulse function with a period of $2 \mathrm{~S}$, amplitude of $2 \mathrm{~V}$, time delay of $0.7 \mathrm{~S}$, and pulse width $30 \%$ of period.

The motor voltages behave well under the maximum permitted value of $40 \mathrm{~V}$ as shown in figure 6 . The effects of disturbances in tracking performance are represented in figure 7. 


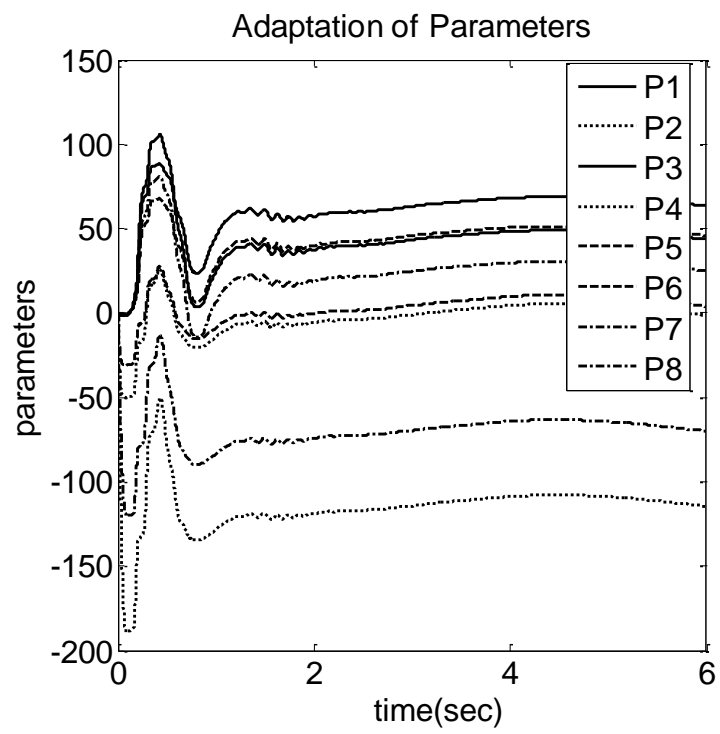

Figure 5. Adaptation of parameters.

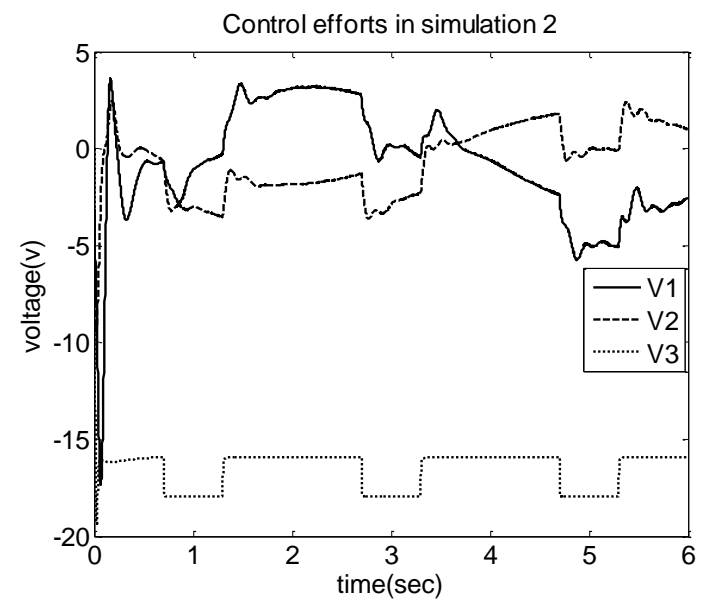

Figure 6. Control efforts in simulation 2 for disturbance rejection.

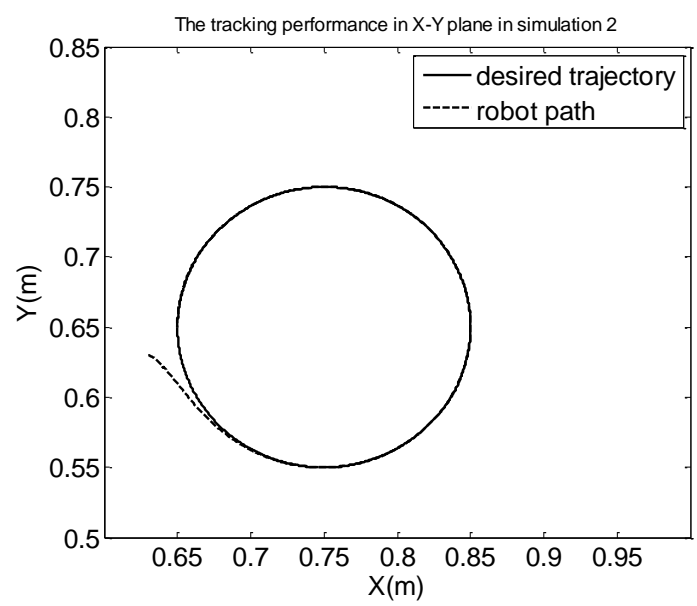

Figure 7. The tracking performance in the $x y$ plane in simulation 2 for disturbance rejection.

\section{Conclusion}

This paper has developed a discrete-time modelfree robust control for electrically driven robot manipulators in task space. The adaptive fuzzy system has estimated the lumped uncertainty very well. The proposed gradient descent algorithm has been able to minimize the estimation error without using any data for the lumped uncertainty. The gradient descent algorithm has performed adaptively to reduce the tracking error in the presence of uncertainties. The proposed method has shown a very good performance such that the value of tracking error are ultimately bounded to small values. The proposed control algorithm has guaranteed the stability analysis and simulation results have shown the effectiveness of the method. The control approach is robust with a very good tracking performance.

\section{References}

[1] Ogata, K. (1987). Discrete-Time Control Systems. Prentice-Hall, NJ.

[2] Lam, H. K. \& Leung, F. H. F. (2008). Stability analysis of discrete-time fuzzy-model-based control systems with time delay: Time delay-independent approach. Fuzzy Sets and Systems, vol. 159, no. 8, pp. 990-1000.

[3] Wu, L., Su, X., Shi, P. \& Qiu, J. (2011). A new approach to stability analysis and stabilization of discrete-time T-S fuzzy time-varying delay systems. IEEE Transactions on Systems, vol. 41, no. 1, pp. 273286.

[4] Lee, W. K., Hyun, C. H., Lee, H., Kim, E. \& Park, M. (2007). Model reference adaptive synchronization of T-S fuzzy discrete chaotic systems using output tracking control. Chaos, Solitons and Fractals, vol. 34, no. 5 , pp. $1590-1598$.

[5] Kempf, C., Messner, W., Tornizuka, M. \& Horowitz, R. (1993). Comparison of four discrete-time repetitive control algorithms. IEEE Cont Syst Mag, vol. 13 , no. 6, pp. 48-54.

[6] Shoja Majidabad, S. \& Shandiz, H. T. (2012). Discrete-time based sliding-mode control of robot manipulators. International Journal of Intelligent Computing and Cybernetics. vol. 5, no. 3, pp. 340-358.

[7] Fateh, M. M., Azargoshasb, S. \& Khorashadizadeh, S. (2013). A model-free discrete control for robot manipulators using a fuzzy estimator. The International Journal for Computation and Mathematics in Electrical and Electronic Engineering, Accepted 1-Nov-2013.

[8] Liu, C., Cheah, C. C. \& Slotine, J. J. E. (2006). Adaptive Jacobian tracking control of rigid-link electrically driven robots based on visual task-space information. Automatica, vol. 42, no. 9, pp. 1491-1501.

[9] Cheah, C. C., Liu, C. \& Slotine, J. J. E. (2006). Adaptive Jacobian tracking control of robots with uncertainties in kinematic, dynamic and actuator models. IEEE Trans. Automatic control. vol. 51, no. 6, pp. 1024-1029. 
[10] Sage, H. G., De Mathelin, M. F. \& Ostertag, E. (1999). Robust control of robot manipulators. a survey. Int. J. Control, vol. 72, no. 16, pp. 1498-1522.

[11] Fateh, M. M. (2010). Robust voltage control of electrical manipulators in task-space. Int. J. Innov. Comput. Info. Control, vol. 6, no. 6, pp. 2691-2700.

[12] Fateh, M. M. (2012). Nonlinear control of electrical flexible-joint robots. Nonlinear Dyn. vol. 67, no. 4, pp. 2549-2559.

[13] Fateh, M. M. (2012). Robust control of flexiblejoint robots using voltage control strategy. Nonlinear Dyn. vol. 67, no. 2, pp. 1525-1537.

[14] Wai, R. J. \& Muthusamy, R. (2013). Fuzzyneural-network inherited sliding-mode control for robot manipulator including actuator dynamics. IEEE Transactions on Neural Networks and Learning Systems, vol. 24, no. 2, pp. 274-287.

[15] Yoo, B. K. \& Ham, W. C. (2000). Adaptive control of robot manipulator using fuzzy compensator. IEEE Transactions on Fuzzy Systems, vol. 8, no. 2, pp. 186-199.

[16] Wang, L. X. (1994). Adaptive fuzzy systems and control. Prentice Hall.

[17] Feng, G. \& Chen, G. (2005). Adaptive control of discrete-time chaotic systems: a fuzzy control approach. Chaos, Solitons and Fractals, vol. 23, no. 2, pp. 459-467.
[18] Erbatur, K., Kaynak, O., Sabanovic, A. \& Rudas, I. (1996). Fuzzy adaptive sliding mode control of a direct drive robot. Robotics and Autonomous Systems, vol. 19 , no. 2 , pp. 215-227.

[19] Qu, Z. \& Dawson, D.M. (1996). Robust tracking control of robot manipulators. IEEE Press, Inc., New York.

[20] Talole, S. E. \& Phadke, S. B. (2008). Model following sliding mode control based on uncertainty and disturbance estimator. Journal of Dynamic Systems, Measurement, and Control, vol. 130, no. 3, pp. 1-5.

[21] Fateh, M. M. (2010). Proper uncertainty bound parameter to robust control of electrical manipulators using nominal model. Nonlinear Dynamics, vol. 61, no. 4, pp. 655-666.

[22] Spong, M. W., Hutchinson, S. \& Vidyasagar, M. (2006). Robot Modelling and Control. Wiley, Hoboken.

[23] Wang, L. X. (1997). A Course in Fuzzy Systems and Control. Prentice-Hall, New York.

[24] Fateh, M. M. \& Babaghasabha, R. (2010). Impedance control of robots using voltage control strategy. Nonlinear Dynamics, Accepted 19 May 2013. 
كنترل مقاوم زمان كسسته بازوى مكانيكى ربات در فضاى كار با استفاده ازتخمينكر فازى تطبيقى

محمدمهدى فاتح" و سيامك آذركشسب

كروه كنترل، دانشكده مهندسى برق، دانشكاه صنعتى شاهرود، شاهرود، ايران.

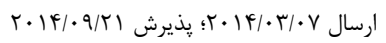

جكيده:

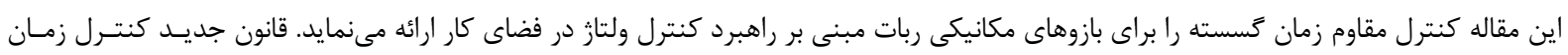

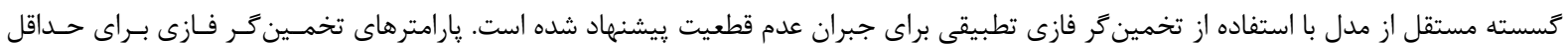

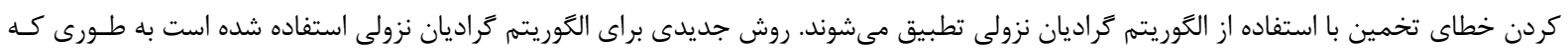

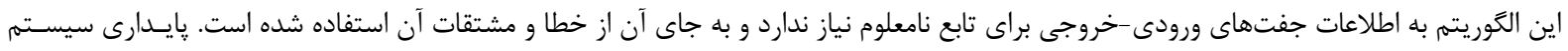

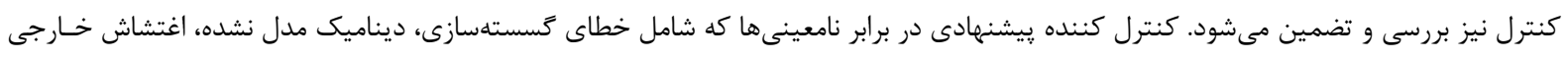

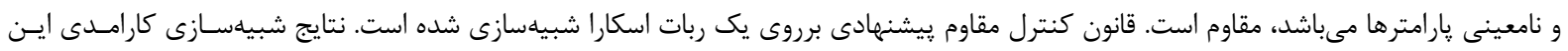
روش كنترلى را نشان مى دهد. 